\title{
Machine learning: Hit Time Finding with a Neural Network
}

\author{
R. Thalmeier ${ }^{b, *, *}$, H. Yin ${ }^{b, *}$, H. Aihara ${ }^{q}$, T. Aziz ${ }^{i}$, S. Bacher ${ }^{u}$, S. Bahinipati ${ }^{d}$, E. Barberio ${ }^{a}$, Ti. Baroncelli $^{a}$, To. $^{2}$ \\ Baroncelli $^{a}$, A. K. Basith ${ }^{e}$, G. Batignani ${ }^{j, k}$, A. Bauer ${ }^{b}$, P. K. Behera ${ }^{e}$, V. Bertacchi $\mathbf{i}^{j, k}$, S. Bettarini ${ }^{j, k}$, B. Bhuyan ${ }^{f}, \mathbf{T}$. \\ Bilka $^{c}$, F. Bosi ${ }^{k}$, L. Bosisio ${ }^{l, m}$, A. Bozek ${ }^{u}$, F. Buchsteiner ${ }^{b}$, G. Caria ${ }^{a}$, G. Casarosa $^{j, k}$, M. Ceccanti $^{k}$, D. Cervenkov ${ }^{c}$, T. \\ Czank $^{p}$, N. Dash ${ }^{d}$, M. De Nuccio ${ }^{j, k}$, Z. Doležal ${ }^{c}$, F. Forti ${ }^{j}, k$, M. Friedl ${ }^{b}$, B. Gobbo ${ }^{m}$, J. A. M. Grimaldo ${ }^{q}$, K. Harar ${ }^{r}$, T. \\ Higuchi $^{n}$, C. Irmler ${ }^{b}$, A. Ishikawa ${ }^{p}$, H. B. Jeon ${ }^{s}$, C. Joo ${ }^{n}$, M. Kaleta ${ }^{u}$, J. Kandra ${ }^{c}$, N. Kambara ${ }^{r}$, K. H. Kang ${ }^{s}$, P. \\ Kodys $^{c}$, T. Kohriki ${ }^{r}$, S. Koike ${ }^{C, r}$, I. Komarov ${ }^{m}$, M. Kumar ${ }^{g}$, R. Kumar ${ }^{h}$, W. Kun ${ }^{q}$, P. Kvasnicka ${ }^{c}$, C. La Licata ${ }^{l, m}$, K. \\ Lalwani $^{g}$, L. Lanceri ${ }^{l, m}$, J. Y. Lee ${ }^{t}$, S. C. Lee ${ }^{s}$, J. Libby ${ }^{e}$, T. Lueck ${ }^{j, k}$, P. Mammini ${ }^{k}$, A. Martini ${ }^{j, k}$, S. N. Mayekar ${ }^{i}$, G. B. \\ Mohanty $^{i}$, T. Morii ${ }^{n}$, K. R. Nakamura ${ }^{r}$, Z. Natkaniec ${ }^{u}$, Y. Onuki ${ }^{q}$, W. Ostrowicz ${ }^{u}$, A. Paladino ${ }^{n}$, E. Paoloni ${ }^{j}, k$, H. Park ${ }^{s}$, \\ K. Prasanth $^{i}$, A. Profeti ${ }^{k}$, I. Rashevskaya ${ }^{A, m}$, K. K. Rao ${ }^{i}$, G. Rizzo ${ }^{j, k}$, Resmi P. K. $^{e}$, M. Rozanska ${ }^{i}$, D. Sahoo ${ }^{i}$, J. \\ Sasaki $^{q}$, N. Sato ${ }^{r}$, S. Schultschik ${ }^{b}$, C. Schwanda ${ }^{b}$, J. Stypula ${ }^{u}$, J. Suzuki ${ }^{r}$, S. Tanaka ${ }^{r}$, H. Tanigawa ${ }^{q}$, G. N. Taylor ${ }^{a}$, \\ T. Tsuboyama ${ }^{r}$, P. Urquijo ${ }^{a}$, L. Vitale ${ }^{l, m}$, S. Watanuki ${ }^{p}$, M. Watanabe ${ }^{B, o}$, I. J. Watson ${ }^{q}$, J. Webb $^{a}$, J. Wiechczynski ${ }^{u}, \mathbf{S}$. \\ Williams $^{a}$, L. Zani ${ }^{j, k}$ (Belle-II SVD Collaboration) \\ a School of Physics, University of Melbourne, Melbourne, Victoria 3010, Australia \\ ${ }^{b}$ Institute of High Energy Physics, Austrian Academy of Sciences, 1050 Vienna, Austria \\ ${ }^{c}$ Faculty of Mathematics and Physics, Charles University, 12116 Prague, Czech Republic \\ ${ }^{d}$ Indian Institute of Technology Bhubaneswar, Satya Nagar, India \\ ${ }^{e}$ Indian Institute of Technology Madras, Chennai 600036, India \\ ${ }^{f}$ Indian Institute of Technology Guwahati, Assam 781039, India \\ ${ }^{g}$ Malaviya National Institute of Technology Jaipur, Jaipur 302017, India \\ ${ }^{h}$ Punjab Agricultural University, Ludhiana 141004, India \\ ${ }^{i}$ Tata Institute of Fundamental Research, Mumbai 400005, India \\ ${ }^{j}$ Dipartimento di Fisica, Università di Pisa, I-56127 Pisa, Italy \\ ${ }^{k}$ INFN Sezione di Pisa, I-56127 Pisa, Italy \\ ${ }^{l}$ Dipartimento di Fisica, Università di Trieste, I-34127 Trieste, Italy \\ ${ }^{m}$ INFN Sezione di Trieste, I-34127 Trieste, Italy, ${ }^{A}$ presently at TIFPA - INFN, I-38123 Trento, Italy \\ ${ }^{n}$ Kavli Institute for the Physics and Mathematics of the Universe (WPI), University of Tokyo, Kashiwa 277-8583, Japan \\ ${ }^{o}$ Department of Physics, Niigata University, Niigata 950-2181, Japan, ${ }^{B}$ presently at Nippon Dental University, Niigata \\ 951-8580, Japan \\ ${ }^{p}$ Department of Physics, Tohoku University, Sendai 980-8578, Japan \\ ${ }^{q}$ Department of Physics, University of Tokyo, Tokyo 113-0033, Japan \\ ${ }^{r}$ High Energy Accelerator Research Organization (KEK), Tsukuba 305-0801, Japan, ${ }^{C}$ deceased \\ ${ }^{s}$ Department of Physics, Kyungpook National University, Daegu 702-701, Korea \\ ${ }^{t}$ Department of Physics and Astronomy, Seoul National University, Seoul 151-742, Korea \\ ${ }^{u}$ H. Niewodniczanski Institute of Nuclear Physics, Krakow 31-342, Poland
}

EMails: Richard.Thalmeierdoeaw.ac.at, Hao.Yin@oeaw.ac.at

At the High Energy Accelerator Research Organization (KEK) in Tsukuba, Japan, the doublesided silicon strip sub-detector of the Belle II experiment is read out by 1748 APV25 chips. FPGAs perform several calculations on the digitized signals. One of them will be "Hit Time Finding": the determination of the time and amplitude of the signal peaks of each event in real time using pre-programmed neural networks. This work analyses the possibility, precision and reliability of these calculations depending on various parameters.

Topical Workshop on Electronics for Particle Physics (TWEPP2018)

17-21 September 2018

Antwerp, Belgium

* Speaker. $\quad * *$ First authors. 


\section{Introduction}

At the High Energy Accelerator Research Organization (KEK) in Tsukuba, Japan, the Belle II experiment [1] will explore the asymmetry between matter and antimatter and search for new physics beyond the standard model. One of its inner tracking systems is the Silicon Vertex Detector (figure 1) [2], which consists of 172 orthogonal double-sided strip sensors. They are arranged cylindrically in four layers around the Pixel Detector to measure the tracks of the collision products of electrons and positrons.

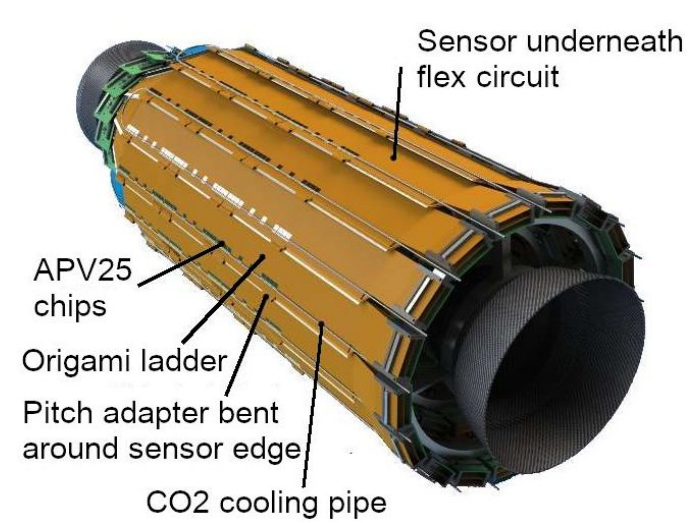

Figure 1: The Belle II Detector

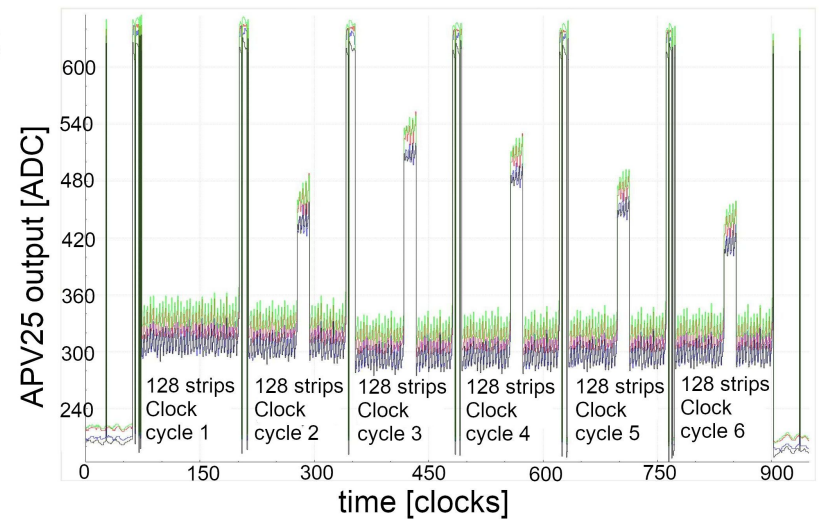

Figure 2: APV25 calibration pulse output

These sensors are read out by 1748 APV25 chips [3], each for 128 silicon strips. They have a shaping time of about $50 \mathrm{~ns}$ and output a time-multiplexed signal for each event and strip. The analog data are then sent out of the radiation zone to 48 custom-built VME modules which convert them to digital, where 3 or 6 values per event of each strip are sampled at a clock of $31.8 \mathrm{MHz}$. FPGAs then compensate line signal distortions and reflections using digital finite impulse response (FIR) filters and detect data frames from the incoming stream. Then they perform pedestal subtraction, common mode correction and zero suppression. And finally "Hit Time Finding", which will be implemented according to the findings below into the FPGA firmware [4]: the determination of time and amplitude of the signal peaks of three selected samples of the APV25 outputs (figure 2) of each event on each silicon strip in real time using pre-trained neural networks. Shown in this paper are preliminary studies to explore the possibility to implement a hit time finding algorithm in FPGAs .

\section{The Hit Time Finding Network}

The APV25 has a so-called internal calibration circuit that is able to inject a configurable amount of charge into each pre-amplifier. The injected signal can be delayed in steps of $1 / 8$ th of a clock cycle $(\sim 3.9 \mathrm{~ns})$. By scanning those parameters and trigger latency we can probe the calibration pulse at various timings and amplitudes (figure 3). The gray lines mark the training range, where the first frame signal is located at; the two following consecutive signals are $\sim 32 \mathrm{~ns}$ apart. For the neural network [5] training we opted to use the averaged calibration pulse. Single strip pulses are used to verify the range of the output in order to check for over training. Figure 5 
shows this hit time (T) / amplitude (A) finding network, an additional verification network, which re-calculates optimal three APV25 samples for the given hit time and amplitude, and the algorithm for the control flow.

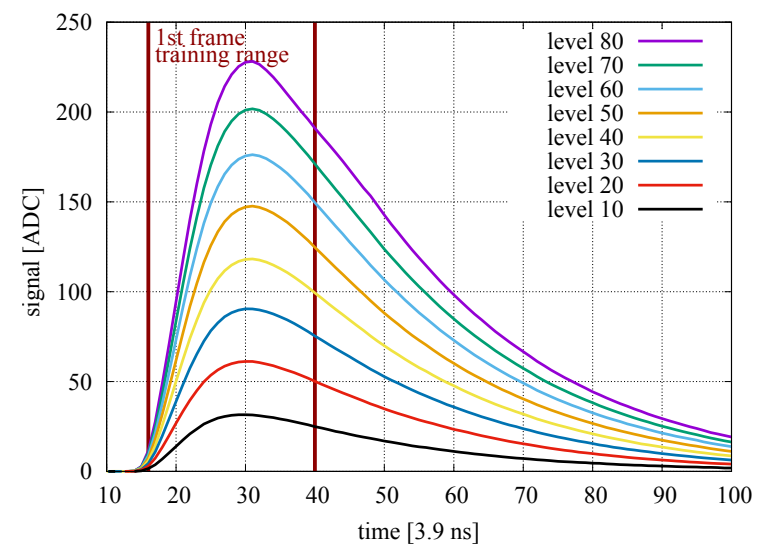

Figure 3: APV25 calibration pulses with different amplitudes

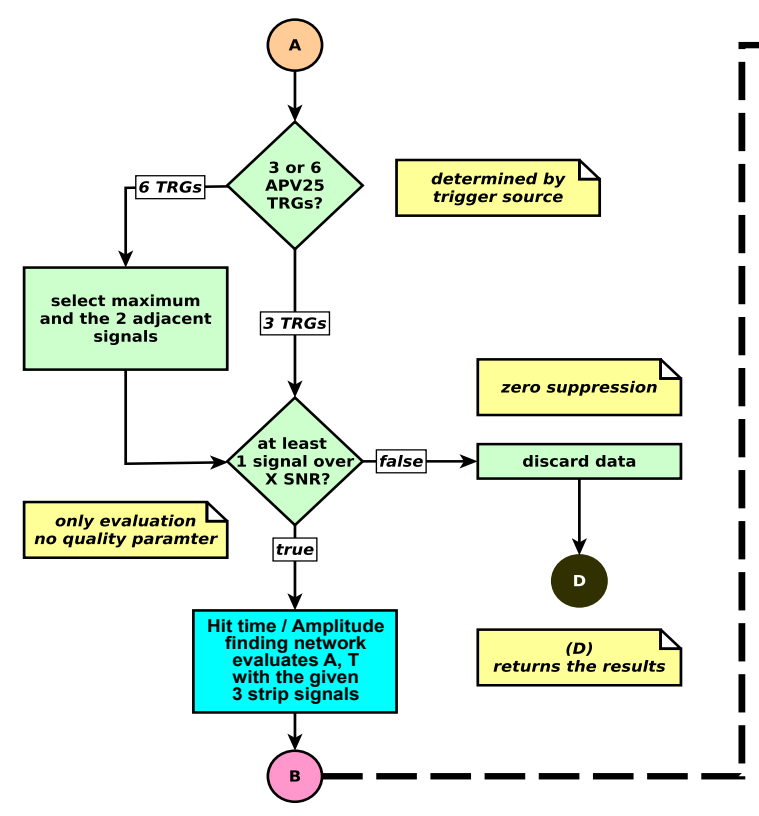

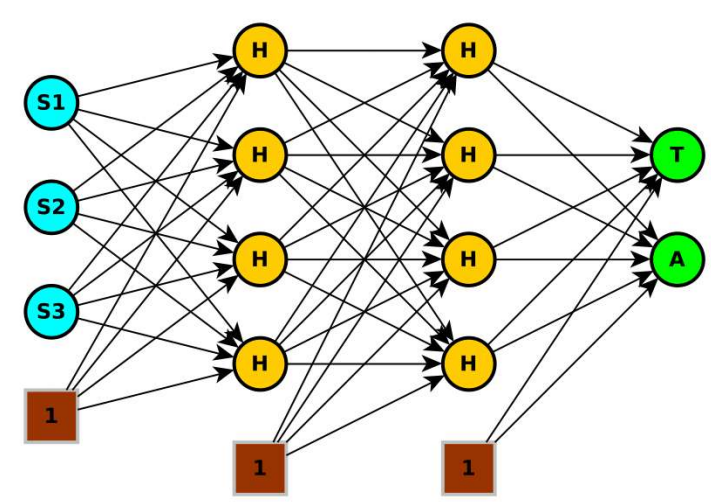

Figure 4: Neural network layout for hit time and amplitude

Figure 5: Control Flow

Both networks are optimized using online training: at each sample the weights are adjusted. The final accuracy of both networks is strongly dependent on the layout, see figure 6 and 7 . With two hidden layers with four nodes each (figure 4), softsign activation functions for the inner layers (efficient FPGA usage) and linear functions for the output layers the precision are sufficient.

Figure 8 shows the accuracy of the hit-time/amplitude network with single strip data. Each strip has a slightly different amplitude, but overall the shapes of the calibration pulses are similar. 


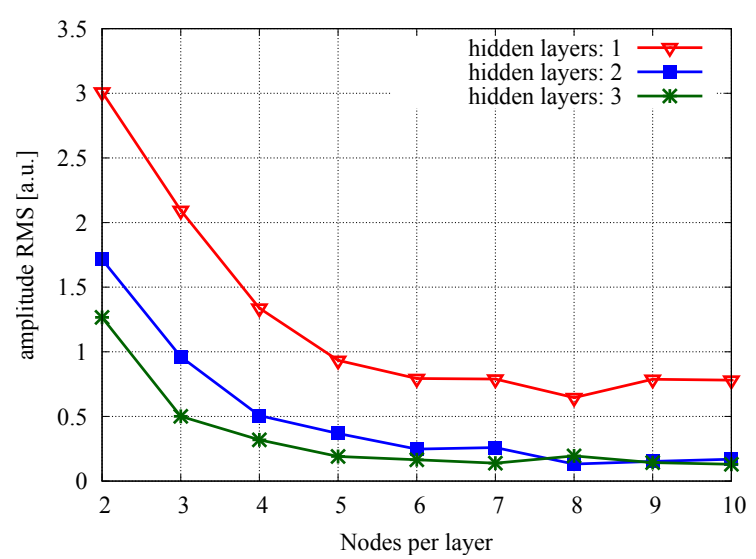

Figure 6: Training error on amplitude using averaged calibration pulse

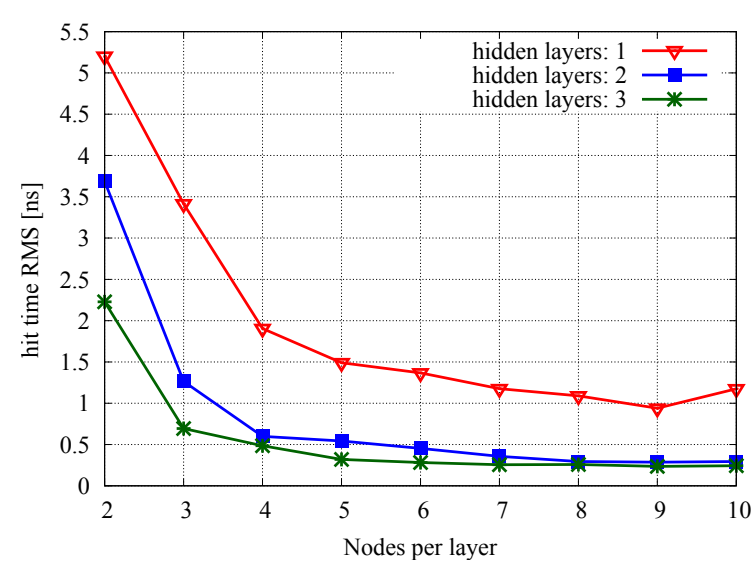

Figure 7: Training error on hit time using averaged calibration pulse

The network is able to precisely estimate the hit time as long as the maximum signal is located at the second frame. However the accuracy drops when the maximum moves to the first frame, which is indicated by the red vertical line in figure 8 . The verification network with hit time and amplitude as input is also able to generate strip signals similar to the training data (figure 9).

Once both networks have been sufficiently trained we applied data taken during BEAST phase 2 with particle collisions.

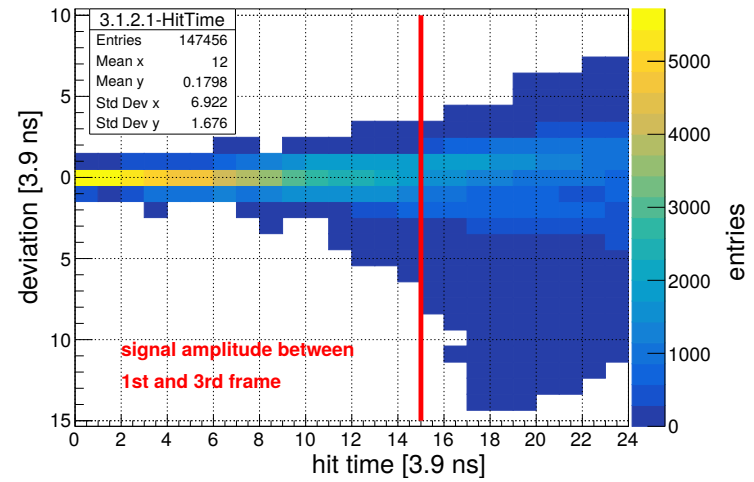

Figure 8: Hit time error after training with averaged calibration pulses

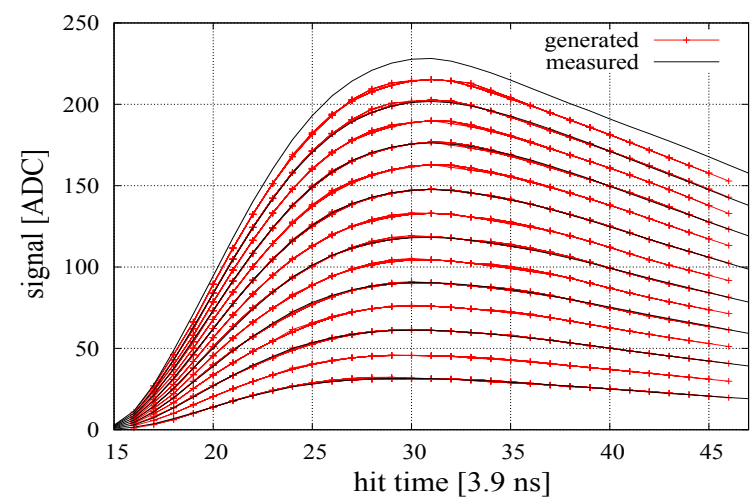

Figure 9: Generated pulses vs averaged calibration pulses

\section{Results}

The verification network shows some deviation between the real-particle pulses and the calibration pulses, whereby the most visible effect has been observed at the lower amplitudes (figure 10); it is also seen in the hit-time distribution (figure 11), but no strong signal shape dependencies are present here. During the phase 2 run number 783 the beam bunches were synchronized with the APV25 clock in such a way that during one clock cycle two distinct bunches were able to collide. Due to trigger jitter we can also see smaller peaks at different trigger arrivals relative to the 


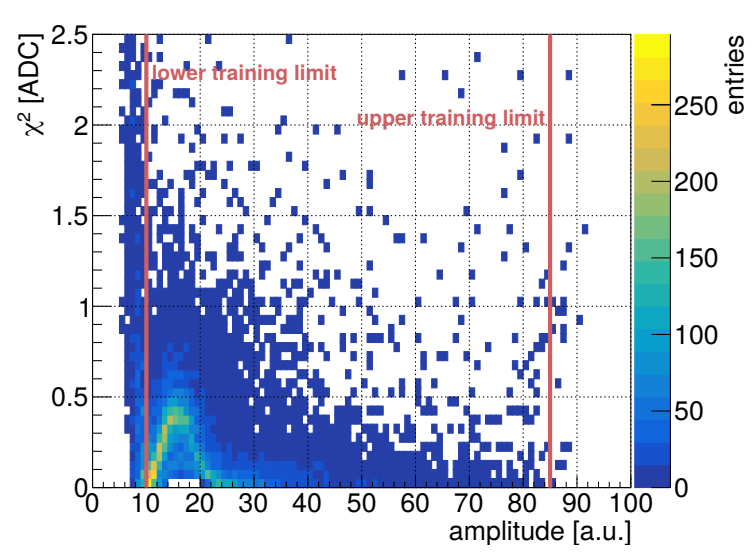

Figure 10: Signal deviations with different amplitudes

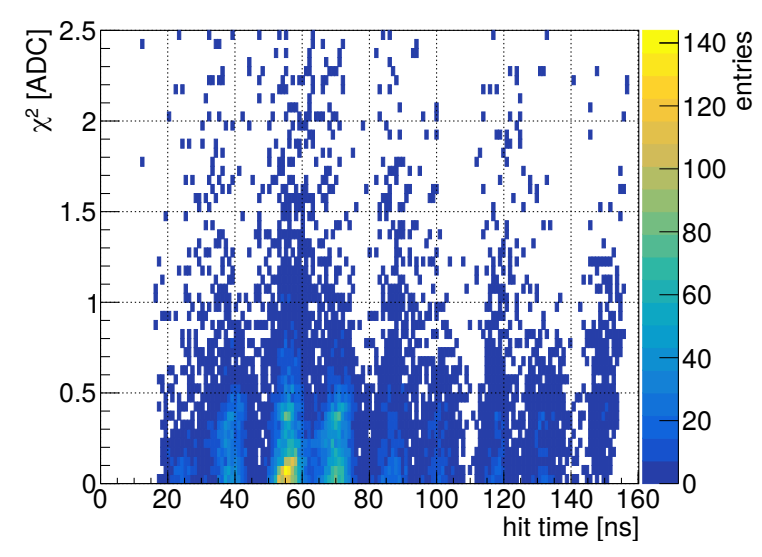

Figure 11: Signal deviations with different hit times

APV25 clock. The distances between each peak show good agreement with the bunch spacing at $\sim 16 \mathrm{~ns}$ (figure 12). The preliminary result also shows good conformity with the offline software analysis, which uses a center-of-gravity algorithm to determine the hit-time distribution (figure 13, which is before time-calibration).

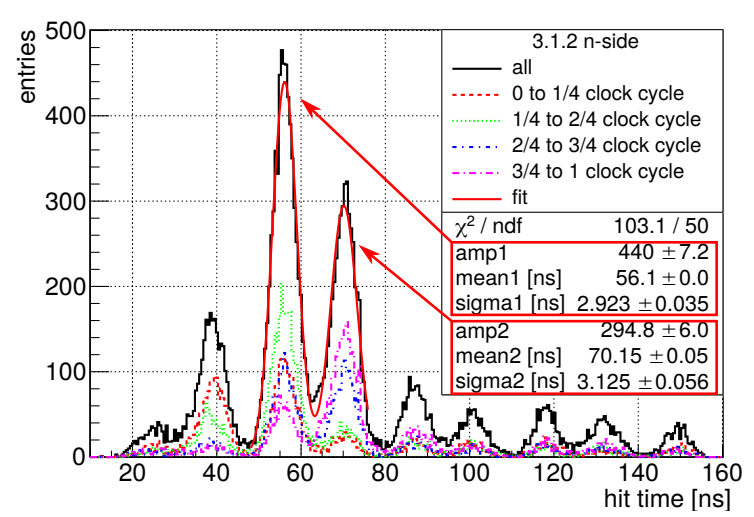

Figure 12: Hit time distribution with particle signals

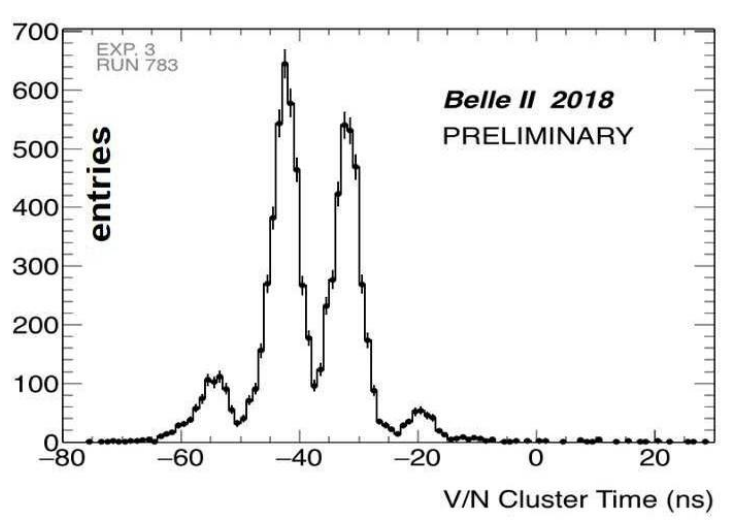

Figure 13: Hit time distribution using center-ofgravity algorithm

\section{References}

[1] BELLE II collaboration, T. Abe, Belle II Technical Design Report, arXiv:1011.0352.

[2] M. Friedl et al., The Belle II Silicon Vertex Detector, Nucl. Instrum. Meth. A 732 (2013) 83.

[3] M. French et al., Design and results from the APV25, a deep sub-micron CMOS front-end chip for the CMS tracker, Nucl. Instrum. Meth. A 466 (2001) 359

[4] R. Thalmeier et al., Electronics and Firmware of the Belle II Silicon Vertex Detector Readout System, PoS TWEPP-17 (2017) 109, https://pos.sissa.it/313/109/pdf

[5] Yu Hen Hu, Jenq-Neng Hwang, Handbook of Neural Network Signal Processing, CRC Press LLC (2002), ISBN 0-8493-2359-2 